\title{
AVALIAÇÃO COMPORTAMENTAL DA ANESTESIA POR ISOFLUORANO E INFUSÃO CONTÍNUA DE XILAZINA E MEDETOMIDINA EM EQUINOS
}

\author{
Renata Gebara Sampaio Dória ${ }^{1}$; Carlos Augusto Araújo Valadão ${ }^{2}$; Paulo Aléscio \\ CANOla ${ }^{3}$ Érica Cristina Bueno do Prado Guirro ${ }^{4}$; MARINA CECCATO MENDES ${ }^{3}$; ANDré

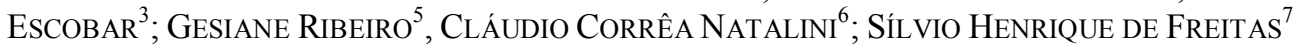 \\ ${ }^{1}$ Professora Doutora do Curso de Medicina Veterinária do Departamento de Zootecnia da Faculdade de Zootecnia e \\ Engenharia de Alimentos (FZEA) da Universidade de São Paulo (USP), Campus de Pirassununga, São Paulo. \\ Jaboticabal, SP. e-mail: redoria@usp.br. Autor para correspondência. \\ ${ }^{2}$ Professor Adjunto, Departamento de Clínica e Cirurgia Veterinária, Universidade Estadual Paulista (UNESP), \\ Faculdade de Ciências Agrárias e Veterinárias (FCAV), Campus de Jaboticabal, SP. \\ ${ }^{3}$ Alunos, Programa de Pós-Graduação, Cirurgia Veterinária, UNESP/FCAV/Jaboticabal. \\ ${ }^{4}$ Professora Doutora,Curso de Medicina Veterinária, Universidade Federal do Paraná, Campus de Palotina, PR. \\ ${ }^{5}$ Professora Doutora,Curso de Medicina Veterinária, Uni-FMU, São Paulo, SP. \\ ${ }^{6}$ Professor Adjunto, Departamento de Farmacologia, Universidade Federal do Rio Grande do Sul, Porto Alegre, RS. \\ ${ }^{7}$ Professor Doutor, Departamento de Clínica e Cirurgia Veterinária, Universidade de Cuiabá (UNIC), Cuiabá, MT.
}

\section{RESUMO}

A associação de um agonista $\alpha-2$ a um protocolo anestésico visa à sedação profunda, miorrelaxamento e analgesia potente, de forma a reduzir a quantidade de outros agentes anestésicos para se alcançar o plano anestésico ideal para a realização de procedimentos cirúrgicos. Neste estudo, avaliou-se comparativamente a qualidade da tranquilização, indução e recuperação da anestesia por isofluorano e infusão contínua de xilazina e medetomidina em 16 equinos e concluiu-se que, nesta espécie animal, o protocolo anestésico com medetomidina garante maior relaxamento muscular, melhor qualidade de indução e recuperação anestésica, embora apresente a mesma qualidade de tranquilização que a xilazina.

PALAVRAS-CHAVE: agonistas $\alpha-2$; comportamento; equinos; infusão contínua; isofluorano.

\section{BEHAVIORAL EVALUATION OF THE ISOFLURANE ANESTHESIA AND XYLAZINE AND MEDETOMIDINE CONTINUOUS INFUSION IN HORSES}

\section{ABSTRACT}

The association of an $\alpha-2$ agonist to an anesthetic protocol leads to deep sedation, muscle relaxation and potent analgesia, reducing the quantity of other anesthetic agents to reach the ideal anesthetic plan to the surgical procedure. This study evaluated comparatively the tranquilization, induction and recovery quality of the isofluorane anesthesia and xylazine and medetomidine continuous infusion in 16 horses. It was possible to conclude that, in this specie, the medetomidine protocol warrants wider muscle relaxation, better quality of anesthetic induction and recovery, even though it presents the same tranquilization quality of the xylazine protocol.

KEYWORDS: egg quality; glutamine; intestinal morphology; thermal stress. 


\section{INTRODUÇÃO}

A associação de anestésicos inalatórios a fármacos tranquilizantes injetáveis, adotada recentemente, tem sido considerada a melhor maneira de se reduzir a concentração dos agentes anestésicos inalatórios, tornando, certamente, mais seguro o procedimento anestésico. Os benefícios incluem melhor controle da profundidade anestésica e, consequentemente, a recuperação anestésica pode vir a ser mais rápida, controlada e tranquila quando comparada ao uso isolado de anestésicos inalatórios, uma vez que esta é determinada, principalmente, pelo perfil farmacocinético dos agentes empregados (NOLAN \& HALL, 1985; EWING et al., 1993; BETTSCHART-WOLFENSBERGER et al., 2001; MUIR, 2004; KUSHIRO et al., 2005).

Embora a utilização de agonistas $\alpha-2$ não seja totalmente livre de preocupações relacionadas com seus efeitos cardiovasculares, as vantagens de se associar um agonista $\alpha-2$ ao protocolo anestésico envolvem sedação profunda, miorrelaxamento e analgesia potentes que reduzem a quantidade de outros agentes anestésicos para se alcançar o plano anestésico ideal para a realização de procedimentos cirúrgicos (MURRELL \& HELLEBREKERS, 2005).

A xilazina e a medetomidina são substâncias com propriedades sedativa e analgésica, altamente lipofílicas, com volume de distribuição alto, cuja meia vida de eliminação situa-se entre 30 a 90 minutos (min) e de clearance corpóreo total entre 20 e $80 \mathrm{~mL} / \mathrm{kg} / \mathrm{min}$, classificadas farmacologicamente como agonistas dos receptores adrenérgicos do tipo $\alpha-2$ (agonista $\alpha-2)$ (SALONEN, 1992).

Clinicamente, em equinos, a xilazina causa sedação máxima após três a cinco minutos da administração intravenosa, durando cerca de 30 a 40 minutos. É utilizada em associação com a cetamina para produzir anestesia de curta duração, com analgesia e efeitos colaterais mínimos, garantindo uma recuperação anestésica tranquila (LIN, 1996).

A medetomidina, embora similar à xilazina, produz efeitos comportamentais mais potentes (WAGNER et al., 1991; BRYANT et al., 1996). Sugere-se que, embora possua um perfil farmacodinâmico similar aos outros agonistas $\alpha-2$ empregados em equinos, a medetomidina promova maior analgesia, sedação e ataxia que a xilazina devido, possivelmente, à penetração mais rápida no sistema nervoso central (SNC)
(KAMERLING et al., 1991; BUENO et al., 1999), contribuindo para isso a elevada seletividade da medetomidina aos receptores $\alpha-2$, ou seja, seletividade de 1620 se comparada com a da xilazina de 160 (THURMON et al., 1996). Clinicamente, os equinos tornam-se sedados e irresponsivos ao ambiente de dois a sete minutos após administração intravenosa única de medetomidina e esse efeito permanece inalterado durante 20 a 30 minutos. Dessa forma, se este bolus inicial for associado à infusão contínua de medetomidina, o nível plasmático desse fármaco torna-se estável em 30 minutos e essa estabilidade permanece durante todo o período de infusão (BETTSCHART-WOLFENSBERGER et al.,1999).

A medetomidina tem mostrado ser segura para ser utilizada em esquemas de infusão contínua, em equinos, uma vez que em taxa de infusão adequada produz sedação prolongada relacionada à manutenção de concentração plasmática estável. Na velocidade de infusão de $3,5 \mu \mathrm{g} / \mathrm{kg} / \mathrm{h}$ promove um efeito sedativo constante, analgesia e mínimos efeitos cardiopulmonares (BETTSCHARTWOLFENSBERGER et al., 2001).

O midazolam é um agente sedativo de curta duração, com uma rápida meia-vida de eliminação e um clearance corpóreo total rápido, sendo que sua concentração plasmática atinge valores abaixo do limite de detecção 20 minutos após início de administração (JOHNSON et al., 2003). Tem sido utilizado em doses baixas para aumentar a sedação e prevenir hipertonia muscular. Em equinos, o midazolam tem sido indicado, em associação com a cetamina, para induzir anestesia geral, mas podem resultar em fraqueza, fasciculação muscular e ataxia (THURMON et al., 1996).

A cetamina é um agente anestésico dissociativo de elevada lipossolubilidade, tornando o SNC incapaz de receber ou processar informações sensoriais e, consequentemente, modular a resposta aos estímulos nocivos. Os resultados da administração de cetamina são anestesia, analgesia, amnésia, supressão do medo e da ansiedade (BERGMAN, 1999). A cetamina induz efeito anestésico imediato, em menos de um minuto, após administração intravenosa, em equinos (KAKA et al., 1979). Possui fase de distribuição rápida, cuja meia-vida é de aproximadamente três minutos e a concentração plasmática mínima é alcançada, aproximadamente, 12 minutos após sua administração (KAKA et al., 1979; WATERMAN 
et al., 1987).

A cetamina é distribuída comercialmente na forma racêmica, constituída por dois isômeros: $50 \% \quad \mathrm{R}(-)$ e $50 \% \quad \mathrm{~S}(+)$. As propriedades farmacocinéticas desses dois compostos praticamente não variam, exceto que o clearance plasmático da cetamina $\mathrm{S}(+)$ é mais rápido que o da R(-). Dessa forma, a recuperação anestésica é mais curta. Os efeitos psicotrópicos adversos têm sido atribuídos ao isômero $\mathrm{R}(-)$, enquanto que o isômero $\mathrm{S}(+)$, mesmo em doses hipnóticas, tem mostrado menor incidência de efeitos indesejáveis que o isômero R(-) (DUQUE et al., 2004). O isômero $\mathrm{S}(+)$ induz hipnose que dura, aproximadamente, duas vezes mais que o $\mathrm{R}(-)$, com analgesia mais profunda e menor atividade locomotora durante a recuperação anestésica (LIN, 1996). O isômero $\mathrm{S}(+)$ demonstrou possuir uma estereoseletividade, através do bloqueio não competitivo dos receptores NMDA; potências anestésica, analgésica e hipnótica dois a quatro vezes maior que a do isômero $\mathrm{R}(-)$; índice terapêutico duas vezes e meia maior que o da forma $\mathrm{R}(-)$ e sendo a dose efetiva (DE50) significativamente menor (DUQUE et al., 2004). Por essa razão preconiza-se, em estudos clínicos, a redução de 50 a $70 \%$ na dose do isômero $\mathrm{S}(+)$, em relação à dose recomendada para o racemato (KIENBAUM et al., 2001).

Dentre os anestésicos voláteis, o isofluorano é um anestésico inalatório amplamente utilizado, pertencente ao grupo dos halogenados. Possui reduzido coeficiente de solubilidade sangue/gás $(1,46)$ e, devido a isso, a indução, recuperação e respostas às mudanças nas concentrações de isofluorano ocorrem rapidamente (STEFFEY, 1996). O isofluorano é minimamente metabolizado pelos animais, sendo que, em média, $94 \%$ ou mais do isofluorano é exalado inalterado. Em equinos, a concentração de isofluorano no ar expirado é reduzida, após três minutos de sua retirada, em $50 \%$, e após 25 minutos, em 90\% (SUMMARY OF PRODUCT CHARACTERISTICS - ISOCARE).

Sendo assim, neste trabalho avaliou-se comparativamente a qualidade da tranquilização, indução e recuperação da anestesia por isofluorano e infusão contínua de xilazina e medetomidina, em equinos.

\section{MATERIAL E MÉTODOS}

Foram utilizados oito equinos adultos, sem raça definida, considerados, após exames clínicos e laboratoriais, hígidos, com peso corpóreo médio de $400 \pm 26 \mathrm{~kg}$ (variando entre
$357 \mathrm{~kg}$ e $465 \mathrm{~kg}$ ), distribuídos em dois grupos experimentais (Grupo Xilazina - GX - e Grupo Medetomidina - GM), de oito animais cada. Os mesmos animais participaram dos dois grupos experimentais, respeitando-se um intervalo de pelo menos um mês entre cada procedimento. Este estudo foi aprovado pela Comissão de Ética e do Bem-Estar dos Animais (CEBEA) do Campus de Jaboticabal da UNESP, conforme protocolo $\mathrm{n}^{\circ}$ 004499-05.

Após jejum alimentar doze horas antes do início do experimento, os equinos de ambos os grupos receberam medicação pré-anestésica, cuja administração foi padronizada em um minuto, sendo, no GX, midazolam ${ }^{1}[0,1 \mathrm{mg} / \mathrm{kg}$, via intravenosa (IV)] e xilazina ${ }^{2}(0,75 \mathrm{mg} / \mathrm{kg}$ IV) e, no GM, midazolam $(0,1 \mathrm{mg} / \mathrm{kg}$ IV $)$ e medetomidina $^{3}(7,5 \mu \mathrm{g} / \mathrm{kg}$ IV), acondicionados na mesma seringa; aguardaram-se dez minutos para os animais serem submetidos à indução anestésica com cetamina $\mathrm{S}(+)^{4}(1 \mathrm{mg} / \mathrm{kg} \mathrm{IV})$, cuja administração foi padronizada em 30 segundos. Ato contínuo, os equinos foram intubados com sonda de Magill ${ }^{5}$ compatível com o diâmetro da traquéia, posicionados em decúbito dorsal na mesa cirúrgica, administrando-lhes oxigênio a $100 \%$, com fluxo de $20 \mathrm{~mL} / \mathrm{kg} /$ minuto durante os dez minutos iniciais, seguido de $10 \mathrm{~mL} / \mathrm{kg} /$ minuto, por meio de circuito anestésico semi-fechado ${ }^{6}$, dotado de vaporizador calibrado para isofluorano ${ }^{7}$. Para o fornecimento do anestésico inalatório, a concentração foi ajustada em uma CAM (1,3\%), medida ao final da expiração, pelo analisador de gases anestésicos ${ }^{8}$. Após estabilização em uma CAM de isofluorano, os animais do GX receberam infusão contínua ${ }^{9}$ de $0,35 \mathrm{mg} / \mathrm{kg} / \mathrm{h}$ de xilazina, diluída em solução fisiológica ${ }^{10}$ (diluição $1: 2$ ), e os animais do GM receberam $3,5 \mu \mathrm{g} / \mathrm{kg} / \mathrm{h}$ de medetomidina, diluída em solução fisiológica (diluição 1:10), durante o período de uma hora.

Foram realizadas avaliações da qualidade da tranquilização (Tabela 1) e indução anestésica (Tabela 2), sendo também realizadas avaliações

\footnotetext{
${ }^{1}$ Dormire - Cristália Produtos Químicos e Farmacêuticos Ltda., ItapiraSP, Brasil

${ }^{2}$ Coopazine $2 \%$ - Schering-Plough, Cotia-SP, Brasil

${ }^{3}$ Domitor - Pfizer Animal Health, Inc, Extos, PA, EUA

${ }^{4}$ Ketamin S(+) - Cristália Produtos Químicos e Farmacêuticos Ltda., Itapira-SP, Brasil

${ }^{5}$ Sonda de Magill - Cirurg Fernandes Ltda, São Paulo-SP, Brasil

${ }^{6}$ Aparelho de anestesia inalatória - HB Comercial Hospitalar Ltda, São Paulo-SP, Brasil

${ }^{7}$ Vaporizador calibrado para isofluorano - HB Comercial Hospitalar Ltda, São Paulo-SP, Brasil

${ }^{8}$ OHMEDA mod. 5250, processo FAPESP n ${ }^{\circ}$ 96/12830-0, EUA.

${ }^{9}$ SAGE ATI orion - model 362, EUA.

${ }^{10}$ Fisiológico (Cloreto de Sódio 0,9\%) - JP Indústria Farmacêutica S. A., Riberão Preto-SP, Brasil
} 
complementares à indução. Sendo assim, foram avaliados, após indução anestésica, o relaxamento de musculatura glútea e cervical, a qualidade da intubação traqueal, através da facilidade ou dificuldade em abrir a boca do animal e da presença ou ausência de reflexo de deglutição e movimento de língua, profundidade anestésica e imobilização do animal, o que permitia ser elevado pela talha, e posicionado em decúbito dorsal na mesa cirúrgica. Essas avaliações determinaram a necessidade de complementação da dose anestésica de indução (de 0,5 a $1,0 \mathrm{mg} / \mathrm{kg}$ de cetamina $\mathrm{S}+$ e/ou $15 \mathrm{mg}$ de midazolam) (Tabela 3 ). A qualidade da recuperação anestésica (Tabela 4) e observações complementares, como o tempo para os animais voltarem a apresentar reflexo de deglutição, o tempo para atingirem decúbito esternal, o período de tempo que permaneceram em decúbito esternal, o número de tentativas para levantar e a duração da recuperação anestésica, também foram realizadas.
Tabela 1. Escala proposta para avaliação da tranquilização dos equinos (1-4)

\begin{tabular}{|c|c|}
\hline Escala & Qualidade da Tranqüilização \\
\hline $\boldsymbol{\nabla} 1$ & Animal excitado \\
\hline$\Delta 2$ & Animal levemente excitado \\
\hline$\Delta 3$ & Animal alerta \\
\hline 4 & Animal sedado \\
\hline
\end{tabular}

Tabela 2. Escala proposta para avaliação da indução anestésica dos equinos (1-4)

\begin{tabular}{ll}
\hline Escala Qualidade da Indução Anestésica \\
\hline$\nabla 1$ & $\begin{array}{l}\text { Cai de forma imprevisível com risco de se } \\
\text { machucar }\end{array}$ \\
$\Delta 2$ & $\begin{array}{l}\text { Cai de forma imprevisível, sem risco de se } \\
\text { machucar }\end{array}$ \\
\& 3 & $\begin{array}{l}\text { Deita lentamente, movimentando pernas ou } \\
\text { cabeça } \\
\text { Decúbito lento e progressivo sem pedalar } \\
\text { ou balançar a cabeça }\end{array}$ \\
\hline
\end{tabular}

$\boldsymbol{\nabla}$ Indesejável $\boldsymbol{\Delta}$ Controlável $\odot$ Aceitável 婛Desejável

Tabela 3. Escala proposta para avaliação pós-indução anestésica dos equinos (1-4)

\begin{tabular}{|c|c|c|}
\hline ESCALA & AVALIAÇÃO APÓS INDUÇÃO ANESTÉSICA & DOSE COMPLEMENTAR \\
\hline$\nabla 1$ & $\begin{array}{l}\text { Dificuldade em abrir a boca do animal, presença de } \\
\text { reflexo de deglutição e movimento de língua; animal } \\
\text { em plano anestésico superficial e não permite ser } \\
\text { elevado na talha. }\end{array}$ & $\begin{array}{l}1,0 \mathrm{mg} / \mathrm{kg} \text { de cetamina } \mathrm{S}+\mathrm{e} 15 \mathrm{mg} \\
\text { de midazolam }\end{array}$ \\
\hline$\Delta 2$ & $\begin{array}{l}\text { Dificuldade em abrir a boca do animal, presença de } \\
\text { reflexo de deglutição e movimento de língua, animal } \\
\text { em plano anestésico superficial. }\end{array}$ & $\begin{array}{l}0,5 \mathrm{mg} / \mathrm{kg} \text { de cetamina } \mathrm{S}+\text { e } 15 \mathrm{mg} \\
\text { de midazolam }\end{array}$ \\
\hline $\mathbf{\Delta} 3$ & $\begin{array}{l}\text { Facilidade em abrir a boca do animal, ausência de } \\
\text { reflexo de deglutição e movimento de língua; animal } \\
\text { em plano anestésico superficial e não permite ser } \\
\text { elevado na talha. }\end{array}$ & $0,5 \mathrm{mg} / \mathrm{kg}$ de cetamina $\mathrm{S}+$ \\
\hline 4 & $\begin{array}{l}\text { Facilidade em abrir a boca do animal, ausência de } \\
\text { reflexo de deglutição e movimento de língua, animal } \\
\text { imóvel. }\end{array}$ & Sem dose de indução complementar \\
\hline \multicolumn{3}{|c|}{ VIndesejável $\Delta$ Controlável Desejável } \\
\hline \multicolumn{3}{|c|}{ Tabela 4. Escala proposta para avaliação da recuperação anestésica dos equinos (1-4) } \\
\hline Escala & \multicolumn{2}{|l|}{ Qualidade da Recuperação Anestésica } \\
\hline$\nabla 1$ & \multicolumn{2}{|c|}{ Múltiplas tentativas para levantar; evidente excitação; alto risco de injúrias. } \\
\hline$\Delta 2$ & \multicolumn{2}{|c|}{$\begin{array}{l}\text { Levanta depois de quatro tentativas, após o animal estar posicionado em decúbito esternal, } \\
\text { ataxia significante. }\end{array}$} \\
\hline (-) 3 & \multicolumn{2}{|c|}{ Levanta em duas ou três tentativas, após o animal estar posicionado em decúbito esternal. } \\
\hline 4 & \multicolumn{2}{|c|}{ Levanta na primeira tentativa, após o animal estar posicionado em decúbito esternal. } \\
\hline
\end{tabular}

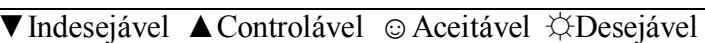


Os dados obtidos na fase experimental foram comparados pelo teste de Mann-Whitney (MannWhitney Rank Sum Test). As diferenças foram consideradas estatisticamente significativas quando $\mathrm{p} \leq 0,05$.

\section{RESULTADOS E DISCUSSÃO}

Recentemente, estabeleceu-se a dose ideal de medetomidina para uso em equinos, baseada na equipotência com a xilazina. YAMASHITA et al. (2000) sugeriram a dose equipotente de medetomidina como sendo $7,5 \mu \mathrm{g} / \mathrm{kg}$ para $1 \mathrm{mg} / \mathrm{kg}$ de xilazina. Segundo BRYANT et al. (1991) e BUENO et al. (1999), a xilazina e a medetomidina possuem equipotência 1:100, em relação a efeitos clínicos e cardiopulmonares. Eles relataram que a diferença nas doses é, provavelmente, dependente da especificidade destes pelos receptores $\alpha$ 2. Dessa forma, neste estudo, a tranquilização foi realizada com doses equipotentes de xilazina e medetomidina, segundo a relação $1: 100(0,75 \mathrm{mg} / \mathrm{kg}$ e $0,0075 \mathrm{mg} / \mathrm{kg}$, respectivamente).

Em relação à qualidade de tranquilização, os dois fármacos mostraram-se eficientes, de forma que, em ambos os grupos, $75 \%$ dos animais encontraram-se sedados após a administração dos fármacos tranquilizantes, não sendo observada diferença significativa entre os grupos (Tabela 5). No GX, dois animais $(25 \%)$ atingiram decúbito esternal cinco e seis minutos após tranquilização. Em ambos os grupos, três animais (37,5\%) apresentaram nistagmo evidente enquanto estavam em posição quadrupedal, fato que se deve ao efeito hipnótico do midazolam, manifestado devido à depressão do sistema límbico (LUNA et al., 1997).

Tabela 5. Valores referentes ao número $\mathrm{e}$ porcentagem (\%) de animais conforme escala préestabelecida para avaliação da qualidade da tranquilização

\begin{tabular}{rcccc}
\hline ESCALA & $\boldsymbol{\nabla} 1$ & $\boldsymbol{\Delta} 2$ & $\boldsymbol{\Delta} 3$ & $\% 4$ \\
\hline GX & 1 & $1(12,5 \%)$ & 0 & 6 \\
& $(12,5 \%)$ & & & $(75 \%)$ \\
GM & 0 & $1(12,5 \%)$ & 1 & 6 \\
& & & $(12,5 \%)$ & $(75 \%)$ \\
\hline
\end{tabular}

Segundo BETTSCHART-WOLFENSBERGER et al. (2001), a dose de $7 \mu \mathrm{g} / \mathrm{kg}$ de medetomidina, antes da injeção de cetamina, promove indução anestésica de boa qualidade e intubação endotraqueal fácil. Esse fato é confirmado neste experimento, pois os equinos pertencentes ao grupo da medetomidina comportaram-se melhor em relação à indução anestésica, sendo que $87,5 \%$ dos animais apresentaram decúbito lento e delicado, sem pedalar ou balançar a cabeça, de acordo com um adequado protocolo de tranquilização e indução. Já nos animais pertencentes ao grupo da xilazina, houve alguns incidentes, como queda imprevisível com risco de se machucar, demonstrando que a medetomidina produz uma tranquilização mais eficiente, embora não tenham sido consideradas significativas as diferenças observadas entre os grupos (Tabela 6).

Tabela 6. Valores referentes ao número e porcentagem $(\%)$ de animais conforme escala préestabelecida para avaliação da qualidade da indução

\begin{tabular}{ccccc}
\hline ESCALA & $\boldsymbol{\nabla} 1$ & $\boldsymbol{\Delta} 2$ & $\odot 3$ & +4 \\
\hline \multirow{2}{*}{$\mathrm{GX}$} & 1 & 0 & 1 & 4 \\
& $(16,7 \%)$ & & $(16,7 \%)$ & $(66,7 \%)$ \\
$\mathrm{GM}$ & 0 & 0 & 1 & 7 \\
& & & $(12,5 \%)$ & $(87,5 \%)$ \\
\hline
\end{tabular}

Ficou demonstrado com este estudo que, em associação com a cetamina $\mathrm{S}(+)$, a medetomidina promove melhor miorrelaxamento $(100 \%$ dos animais apresentaram bom relaxamento da musculatura glútea e cervical após a administração intravenosa em bolus de medetomidina) e melhor qualidade de indução anestésica que a xilazina, de forma que $50 \%$ dos animais não precisaram de complementação medicamentosa à indução anestésica. Por outro lado, $50 \%$ dos cavalos que receberam bolus intravenoso de xilazina não apresentaram relaxamento de musculatura e 100\% necessitaram de doses adicionais de fármacos para concluir a indução anestésica (Tabelas 7 e 8).

Tabela 7. Valores referentes ao número e porcentagem (\%) de animais conforme escala préestabelecida para avaliação do relaxamento de musculatura glútea e cervical

\begin{tabular}{|c|c|c|c|c|}
\hline ESCALA & $\nabla 1$ & $\Delta 2$ & (;) 3 & 4 \\
\hline GX & 0 & $4(50 \%)$ & $\begin{array}{c}3 \\
(37,5 \%)\end{array}$ & $\begin{array}{c}1 \\
(12,5 \%)\end{array}$ \\
\hline $\left.\mathrm{GM}^{(}\right)$ & 0 & 0 & 0 & $\begin{array}{c}8 \\
(100 \%)\end{array}$ \\
\hline
\end{tabular}

Tabela 8. Valores referentes ao número e porcentagem (\%) de animais conforme escala préestabelecida para avaliação complementar à indução

\begin{tabular}{|c|c|c|c|c|}
\hline ESCALA & $\nabla 1$ & $\Delta 2$ & $\Delta 3$ & -4 \\
\hline GX & $\begin{array}{c}5 \\
(62,5 \%)\end{array}$ & 0 & $\begin{array}{c}3 \\
(37,5 \%)\end{array}$ & 0 \\
\hline $\mathrm{GM}^{@}$ & $2(25 \%)$ & $\begin{array}{c}1 \\
(12,5 \%)\end{array}$ & $\begin{array}{c}1 \\
(12,5 \%)\end{array}$ & $\begin{array}{c}4 \\
(50 \%)\end{array}$ \\
\hline
\end{tabular}


@ diferença significativa entre os grupos (teste de MannWhitney, $\mathrm{p} \leq 0,05)$.

Todos os animais do GX e GM permaneceram em um plano superficial de anestesia, evidenciado pela presença de reflexo palpebral característico durante todo o período de avaliação, e, em alguns momentos, lacrimejamento, nistagmo e piscar de pálpebras. Ainda não se sabe se as concentrações plasmáticas de xilazina e medetomidina, especialmente em doses repetidas, estão diretamente correlacionadas à farmacodinâmica, embora os resultados obtidos no estudo de BETTSCHART-WOLFENSBERGER et al. (1999) sugiram que a alteração na taxa de infusão de medetomidina, em pôneis, resulta em mudança no nível plasmático do fármaco e sedação. Da mesma forma, neste estudo, provavelmente, a concentração plasmática reduzida dos agonistas $\alpha-2$, xilazina e medetomidina, foi responsável pela manutenção de um plano anestésico superficial, até mesmo com mobilidade no grupo xilazina. Um dos equinos do GX (12,5\%) não permaneceu imóvel com uma CAM de isofluorano (1,3\%), após 36 minutos de anestesia geral inalatória e infusão contínua de xilazina, necessitando que o anestésico inalatório fosse ajustado até 1,4 CAM $(1,8 \%)$ para que o animal permanecesse imóvel na mesa cirúrgica. Ou seja, o plano anestésico tornou-se superficial demais após redução da concentração plasmática do bolus de xilazina da tranquilização, o que não aconteceu com os animais que receberam medetomidina. Dessa forma, notou-se que a infusão contínua de medetomidina promoveu um plano anestésico mais profundo que a infusão contínua de xilazina, durante anestesia geral inalatória com isofluorano, em equinos.

A recuperação da anestesia é um período de perigo potencial para cavalos e parece ser influenciada por muitos fatores como variabilidade individual, temperamento do animal, presença de hipotensão durante a anestesia, tipo de procedimento cirúrgico, tipo e duração do protocolo anestésico, estímulo externo, utilização de sedativos, ambiente onde o animal vai ser recuperado (YAMASHITA et al., 2000; MATTHEWS et al., 1992; 1998; SANTOS et al., 2003). O isofluorano é um anestésico inalatório considerado seguro (STEFFEY \& HOWLAND, 1980; GROSENBAUGH \& MUIR, 1998; RAISIS et al., 1999), que leva a uma recuperação anestésica rápida (MATTHEWS et al., 1992; DONALDSON et al., 2000; RIPOLL et al., 2000), uma vez que os efeitos depressores cessam rapidamente. Por outro lado, existe uma percepção entre os anestesistas de que a recuperação de anestesia geral inalatória com isofluorano é normalmente agitada e potencialmente violenta (JOHNSTON et al., 2004). Dessa forma, a infusão contínua de agonistas $\alpha-2$ parece ter auxiliado na recuperação anestésica, uma vez que esta, neste experimento, foi tranquila e livre de acidentes. Entre os grupos, observou-se que a qualidade da recuperação anestésica do GM foi superior ao GX (Tabela 9).

Tabela 9. Valores referentes ao número e porcentagem (\%) de animais conforme escala préestabelecida para avaliação da qualidade da recuperação

\begin{tabular}{|c|c|c|c|c|}
\hline ESCALA & $\nabla 1$ & $\Delta 2$ & (:) 3 & 4 \\
\hline GX & $\begin{array}{c}1 \\
(12,5 \%)\end{array}$ & $2(25 \%)$ & $\begin{array}{c}4 \\
(50 \%)\end{array}$ & $\begin{array}{c}1 \\
(12,5 \%)\end{array}$ \\
\hline $\mathrm{GM}^{@}$ & 0 & $\begin{array}{c}1 \\
(12,5 \%)\end{array}$ & $\begin{array}{c}1 \\
(12,5 \%)\end{array}$ & $\begin{array}{c}6 \\
(75 \%)\end{array}$ \\
\hline
\end{tabular}

( diferença significativa entre os grupos (teste de MannWhitney, $\mathrm{p} \leq 0,05)$.

Assim, embora o tempo de recuperação anestésica do grupo sob infusão contínua de medetomidina tenha sido mais prolongado $(29,75 \pm$ 21,56 minutos), 75\% dos animais levantaram em primeira tentativa, após estarem posicionados em decúbito esternal. Neste grupo, os animais apresentaram maior tempo para retornar o reflexo de deglutição $(3,25 \pm 4,37$ minutos $)$ e para se posicionarem em decúbito esternal $(13,14 \pm 4,78$ minutos), permaneceram maior tempo neste decúbito (20,43 $\pm 16,87$ minutos), porém, tentaram se levantar menos vezes $(1,50 \pm 1,07$ minutos $)$ e, então, levantaram-se de maneira adequada, livre de qualquer risco de queda. Já os animais do grupo GX apresentaram uma recuperação anestésica mais rápida $(18,13 \pm 18,48$ minutos), porém de pior qualidade, com menor tempo para retornar o reflexo de deglutição $(1,25 \pm 1,91$ minutos $)$ e para se posicionarem em decúbito esternal $(7,75 \pm 6,82$ minutos), permaneceram menor tempo neste decúbito $(9,75 \pm 13,63$ minutos $)$, aumentando o número de tentativas para levantar $(3,5 \pm 2,07$ minutos) e existindo, embora pequeno, o risco de injúrias. Portanto, não houve sinais físicos de acúmulo de medetomidina ou xilazina, um problema potencial da infusão contínua de agonistas $\alpha-2$, durante anestesia geral inalatória, quando ocorreria aumento na depressão cardiopulmonar induzida pelos anestésicos e resultaria em uma recuperação anestésica demorada e de pobre qualidade (BETTSCHART-WOLFENSBERGER et al., 2001). Dessa forma, este estudo corrobora com os resultados de BETTSCHART-WOLFENSBERGER et al. (2001), propondo que a medetomidina demonstra ser o agonista $\alpha-2$ de escolha para promoção de analgesia intraoperatória, uma vez que mantém a função cardiopulmonar estável e permite uma recuperação rápida e de boa qualidade, mesmo após anestesia prolongada (Tabela 10). 
Tabela 10. Valores médios $\bar{\Upsilon}_{1}$ e desvios padrão $( \pm \mathrm{DP})$, referentes ao tempo para retorno do reflexo de deglutição (min), tempo para alcançar decúbito esternal (min), tempo em posição esternal (min), tentativas para levantar e duração da recuperação $(\mathrm{min})$, de equinos após anestesia com isofluorano sob infusão contínua de xilazina (GX) ou medetomidina (GM)

\begin{tabular}{ccccccc}
\hline GRUPOS & $\begin{array}{c}\text { RETORNO } \\
\text { REFLEXO DE } \\
\text { DEGLUTIÇÃO } \\
(\min )\end{array}$ & $\begin{array}{c}\text { DECÚBITO } \\
\text { ESTERNAL } \\
(\mathrm{min})\end{array}$ & $\begin{array}{c}\text { DURAÇÃO DA } \\
\text { POSIÇÃO } \\
\text { ESTERNAL (min) }\end{array}$ & $\begin{array}{c}\text { TENTATIVAS } \\
\text { PARA }\end{array}$ & $\begin{array}{c}\text { DURAÇÃO DA } \\
\text { RECUPERAÇÃO } \\
(\min )\end{array}$ \\
\hline \multirow{2}{*}{$\mathrm{GX}$} & $\overline{\bar{X}}_{\mathrm{j}}$ & 1,25 & 7,75 & 9,75 & 3,50 & 18,13 \\
& $\pm \mathrm{DP}$ & 1,91 & 6,82 & 13,63 & 2,07 & 18,48 \\
\hline \multirow{2}{*}{$\mathrm{GM}$} & $\overline{\bar{X}}_{\mathrm{j}}$ & 3,25 & 11,5 & $17,9 @$ & 1,50 & $30,12^{@}$ \\
& $\pm \mathrm{DP}$ & 4,37 & 6,41 & 17,21 & 1,07 & 21,63 \\
\hline
\end{tabular}

${ }^{(a)}$ diferença significativa entre os grupos (teste de Mann-Whitney, $\mathrm{p} \leq 0,05$ ).

\section{CONCLUSÕES}

Com base nos resultados obtidos, pode-se concluir que o protocolo anestésico com medetomidina garante maior relaxamento muscular e melhor qualidade de indução anestésica promovida pela cetamina $\mathrm{S}(+)$, embora apresente a mesma qualidade de tranquilização que a xilazina. Além disso, a medetomidina produz uma recuperação anestésica de melhor qualidade, embora mais prolongada que a xilazina.

\section{REFERÊNCIAS}

BERGMAN, A. S. Ketamine: review of its pharmacology and its use in pediatric anesthesia. Anesthesia Progress, v. 46, p. 10-20, 1999.

BETTSCHART-WOLFENSBERGER, R.; CLARKE, K. W.; VAINIO, O.; SHOJAEE ALIABADI, F.; DEMUTH, D. Pharmacokinetics of medetomidine in ponies and elaboration of medetomidine infusion regime which provides a constant level of sedation. Research in Veterinary Science, v. 67, p. 41-46, 1999.

BETTSCHART-WOLFENSBERGER, R.; JÄGGINSCHMUCKER, N.; LENDI, CH.; BETTSCHART, R. W.; CLARKE, K. W. Minimal alveolar concentration of desflurane in combination with an infusion of medetomidine for the anaesthesia of ponies. Veterinary Record, v. 148, p. 264-267, 2001.

BRYANT, C. E.; CLARKE, K.W.; THOMPSON, J. Cardiopulmonary effects of medetomidine in sheep and in ponies. Research in Veterinary Science, v. 60, p. 267-271, 1996.

BRYANT, C. E.; ENGLAND, G.C.W.; CLARKE, K.W. Comparison of the sedative effects of medetomidine and xylazine in horses. Veterinary Record, v. 129, p. 421423, 1991.

BUENO, A. C.; CORNICK-SEAHORN, J.; SEAHORN,
T. L.; HOSGOOD, G.; MOORE, R. M. Cardiopulmonary and sedative effects of intravenous administration of low doses of medetomidine and xylazine to adult horses. American Journal of Veterinary Research, v. 60, p. 1371-1376, 1999.

DONALDSON, L.L.; DUNLOP, G. S.; HOLLAND, M.S.; BURTON, B.A. The recovery of horses from inhalant anesthesia: a comparison of halothane and isoflurane. Veterinary Surgery, v. 29, p. 92-101, 2000.

DUQUE, J. C.; VALADÃO, C.A.A.; FARIAS, A.; ALMEIDA, R.M.; OLESKOVICZ, N. Pre-emptive epidural ketamine or $\mathrm{S}(+)$ ketamine in post-incisional pain in dogs: a comparative study. Veterinary Surgery, v. 33, p. 361-367, 2004.

EWING, K. K.; MOHAMMED, H. O.; SCARLETT, J. M.; SHORT, C. E. Reduction of isoflurane anesthetic requirement by medetomidine and its restoration by atipamezole in dogs. American Journal of Veterinary Research, v. 54, p. 294-299, 1993.

GROSENBAUGH, D.A.; MUIR, W.W. Cardiorespiratory effects of sevoflurane, isoflurane and halothane anesthesia in horses. American Journal of Veterinary Research, v. 59, p. 101-106, 1998.

JOHNSTON, G.M.; EASTMENT, J.K.; TAYLOR, P.M.; WOOD, J.L.N. Is isoflurane safer than halothane in equine anesthesia? Results from a prospective multicentre randomized controlled trial. Equine Veterinary Journal, v. 36, p. 64-71, 2004.

KAKA, J.S.; KLAVANO, P.A.; HAYTON, W.L. Pharmacokinetics of ketamine in the horse. American Journal of Veterinary Research, v. 40, p. 978-81, 1979.

KAMERLING, S.; KEOWEN, M.; BAGWELL, C.; JOCHLE, W. Pharmacological profile of medetomidine in the equine. Acta Veterinaria Scandinavica, v. 87, p.161-162, 1991. Supplementum.

KIENBAUM, P.; HEUTER, T.; PAVLAKOVIC, G.; MICHEL, M.; PETERS, J. S(+) ketamine increases muscle sympathetic activity and maintains the neural 
response to hypotensive challenges in humans. Anesthesiology, v. 94, p. 252-258, 2001.

KUSHIRO, T.; YAMASHITA, K.; UMAR, M. A.; MAEHARA, S.; WAKAIKI, S.; ABE, R.; SENO, T.; TSUZUKI, K.; IZUMISAWA, Y.; MUIR, W. W. Anesthetic and cardiovascular effects of balanced anesthesia using constant rate infusion of midazolamketamine-medetomidine with inhalation of oxygensevoflurane (MKM-OS Anesthesia) in horses. Journal of Veterinary Medical Science, v. 67, p. 379-384, 2005.

LIN, H.C. Dissociative anesthetics. IN: THURMON, J.C.; TRANQUILLI, W.J.; BENSON, G.J. Lumb \& Jones' Veterinary Anesthesia. Baltimore:Williams \& Wilkins. $3^{\mathrm{a}}$ ed. p. 241-296, 1996.

LUNA, S.P.L.; TAYLOR, P.M.; DICK, C.J. Midazolam and ketamine induction before halothane anaesthesia in ponies. Cardiorespiratory, endocrine and metabolic changes. Journal of Veterinary Pharmacology and Therapeutics, v. 20, p. 153-159, 1997.

MATTHEWS, N.S.; MILLER, S.M.; HARTSFIELD, S.M.; SLATER, M.R. Comparison of recoveries from halothane vs. isoflurane anesthesia in horses. Journal of American Veterinary Medical Association, v. 201, p. 559-563, 1992.

MUIR, W.W. New perspectives on the drugs used to produce sedation, analgesia, and anesthesia in horses. IN: Proceedings of the $50^{\text {th }}$ Annual Convention of the American Association of Equine Practitioners, Denver, Colorado, 2004. Lexington: American Association of equine Practitioners. Disponível em: <www.ivis.org $>$. Acesso em 09 jun. 2006.

MURRELL, J.C.; HELLEBREKERS, L.J. Medetomidine and dexmedetomidine: a review of cardiovascular effects and antinociceptive properties in the dog. Veterinary Anaesthesia and Analgesia, v. 32, p. 117-127, 2005.

NOLAN, A. M.; HALL, L.W. Total intravenous anaesthesia in the horse with propofol. Equine Veterinary Journal, v. 17, p. 394-398, 1985.

RAISIS, A.L.; YOUNG, L.E.; BLISSITT, K.J.; BREARLEY, J.C.; MEIRE, H.B.; RANHEIM, B.R.; ARNEMO, J.M.; RYENG, K.A.; SOLI, N.E.; HORSBERG, T.E. A pharmacokinetic study including some relevant clinical effects of medetomidine and atipamizole in lactating dairy cows. Journal of
Veterinary Pharmacology and Therapeutic, v.22, p. 368-373, 1999.

RIPOLL, S.V.; WHITE, K.L.; TAYLOR, P.M. Halotane vs. isoflurane in horses: a retrospective study of 132 cases in a randomized controlled trial. In: Proceedings of the Association of Veterinary Anaesthetists Spring Conference, Cambridge, UK, 2000.

SALONEN, J.S. Chemistry and pharmacokinetics of the $\alpha_{2}$ adrenoceptor agonistis. In: SHORT C. E.; POZNAK, A. Van. Animal Pain. New York: Churchill Livingstone, 1992. p. 191-200.

SANTOS, M.; FUENTE, M.; GARCIA-ITURRALDE, P.; HERRAN, R.; LOPEZ-SANROMAN, J.; TENDILLO, F. J. Effects of alpha-2 adrenoceptor agonists during recovery from isoflurane anaesthesia in horses. Equine Veterinary Journal, v. 35, p. 170-175, 2003.

STEFFEY, E.P. Inhalation anesthetics. IN: THURMON, J.C.; TRANQUILLI, W.J.; BENSON, G.J. Lumb \& Jones' Veterinary Anesthesia. Baltimore:Williams \& Wilkins. $3^{\text {a }}$ ed. p. 183-209, 1996.

SUMMARY OF PRODUCT CHARACTERISTICS ISOCARE (ISOFLURANE, USP), England: Baxter Healthcare, 2004. Disponível em: $<$ http://www.vmd.gov.uk/espcsite/documents/ $>$. Acesso em: 09 jun. 2006.

THURMON, J.C.; TRANQUILLI, W.J.; BENSON, G.J. Preanesthetics and anesthetic adjuncts. IN: _ . Lumb \& Jones' Veterinary Anesthesia. Baltimore:Williams \& Wilkins. $3^{\mathrm{a}}$ ed. p. 183-209, 1996.

WAGNER, A.E.; MUIR, W.W. HINCHCLIFF, K.W. Cardiovascular effects of xylazine and detomidine in horses. American Journal of Veterinary Research, v. 52, p. 651-657, 1991.

WATERMAN, A. E.; ROBERTSON, S.A.; LANE, J.G. Pharmacokinetics of intravenously administered ketamine in the horse. Research in Veterinary Science, v. 42, p. 162-6, 1987.

YAMASHITA, K.; TSUBAKISHITA, S.; FUTAOKA, S.; UEDA, I.; HAMAGUCHI, H.; SENO, T.; KATOH, S.; IZUMISAWA, Y.; KOTANI, T.; MUIR, W.W. Cardiovascular effects of medetomidine, detomidine and xylazine in horses. Journal of Veterinary Medical Science, v. 62, p. 1025-1032, 2000. 